\title{
TEGAFUR ORAL MÁS MITOMICINA VERSUS MITOMICINA INTRAVESICAL SOLA EN LA PROFILAXIS DE LAS RECIDIVAS DE TUMORES VESICALES ESTADIO Ta
}

\author{
G. SERVER PASTOR, M. RIGABERT MONTIEL, V. BAÑÓN PÉREZ, P. VALDELVIRA NADAL, \\ E. CAO AVELLANEDA, J.A. GARCÍA HERNÁNDEZ, M. PÉREZ ALBACETE
}

Servicio de Urología. Hospital Universitario Virgen de La Arrixaca. Murcia.

Actas Urol Esp. 27 (6): 438-341, 2003

\section{RESUMEN}

TEGAFUR ORAL MÁS MITOMICINA VERSUS MITOMICINA INTRAVESICAL SOLA

EN LA PROFILAXIS DE LAS RECIDIVAS DE TUMORES VESICALES ESTADIO Ta

OBJETIVO: Valorar si el empleo de Tegafur oral asociado a Mitomicina intravesical es efectivo en la profilaxis de las recidivas de los tumores vesicales Ta.

MÉTODO: Se trata de un estudio prospectivo en el que comparamos la tasa de recidiva y el tiempo libre de enfermedad de 2 grupos de 40 pacientes cada uno, el primero tratado tras la RTU con Mitomicina intravesical más Tegafur, y el segundo solo con Mitomicina endocavitaria. También se valoró la tolerancia al Tegafur oral.

RESULTADOS: El grupo del Tegafur presentó un descenso de la tasa de recidiva y una prolongación del tiempo libre de enfermedad; pero no resultó estadísticamente significativo. La tolerancia al fármaco fue buena, no apareciendo efectos adversos importantes.

CONCLUSIONES: El Tegafur parece un fármaco útil en la profilaxis de la recidiva de los tumores vesicales superficiales, aunque serán precisos estudios más grandes para sacar conclusiones estadísticamente válidas.

PALABRAS CLAVE: Tegafur. Carcinoma vesical. Quimioterapia.

ABSTRACT

ORAL TEGAFUR AND INTRAVESICAL MITOMYCIN VERSUS INTRAVESICAL MUTOMYCIN ALONE IN THE PROPHYLAXIS OF Ta BLADDER CARCINOMA RECURRENCE

OBJECTIVE: The aim of this study is to know if the use of oral Tegafur associated to intravesical mitomycin is effective in the prevention of the relapses of Ta bladder tumors.

METHOD: This is a prospective study in which we compare the recurrence rate and the diseasefree interval of 2 groups of 40 patients each one, the first of them treated after the TUR with oral Tegafur and intravesical mitomycin, and the second with intravesical mitomycin alone. Tolerance of Tegafur was also studied.

RESULTS: The group of the Tegafur presented a descent of the relapse rate and a continuation of the time free of illness; but it was not statistically significant. The tolerance to the fármaco was good, not appearing important adverse effects.

CONCLUSIONS: Tegafur seems an useful drug in the prevention of the recurrence of superficial bladder tumors, although it will be necessary bigger studies to reach statistically valid conclusions. KEY WORDS: Tegafur. Bladder cancer. Chemotherapy. 
$\mathrm{E}$ la Unión Europea se diagnostican 66.500 nuevos casos de neoplasias vesicales cada año ${ }^{1}$. El $75-80 \%$ de las cuales no infiltrarán el detrusor ${ }^{2}$.

El tratamiento estándar del tumor papilar superficial de vejiga es la resección transuretral. La tasa de recurrencia tras la misma, a los 5 años, oscila entre el 50 y el $90 \%$ de los casos ${ }^{3}$.

Se han descrito varios factores relacionados con un riesgo aumentado de recidiva. En la serie publicada por Meijden et al. ${ }^{4}$ del European Organization for research and treatment of cancer-genitourynary group (EORTC-GU), se identifica como el factor más importante relacionado con la tasa de recurrencia en tumores primarios, el número de tumores; mientras que en tumores recurrentes el factor más importante relacionado con la progresión, la tasa de recurrencia futura y con la muerte por esta enfermedad, es la tasa de recurrencia previa.

La alta tasa y el peligro que entraña la recurrencia de estos tumores, han llevado al empleo de múltiples fármacos para evitarla, con controvertidos resultados y diferente toxicidad local y/o sistémica. Muchas son las líneas de investigación para buscar nuevas sustancias que aumenten la efectividad y disminuyan los efectos secundarios.

El Tegafur es una fluoropirimidina que se administra por vía oral y se activa "in vivo" en el hígado a 5-fluoruracilo, con un espectro antitumoral similar a esta sustancia y mucha menor toxicidad sistémica. Su mecanismo de acción es doble. Por un lado actúa inhibiendo la enzima timidilato-sintetasa, la cual es imprescindible para la sintesis de ADN. Por otro, al metabolizarse a fluoridina-trifosfato y este acoplarse al ARN, lleva a la formación de un ARN anómalo ${ }^{5}$.

Nos planteamos en este estudio investigar la posibilidad de complementar el tratamiento locoregional mediante citostáticos, con otro sistémico de larga duración como el Tegafur, en tumores vesicales superficiales estadio Ta grado citológico I-II sin CIS asociado (los T1, los CIS, los Ta G III, y los Ta con CIS en nuestro protocolo no se tratan con quimioterapia local sino con inmunoterapia local).

\section{MATERIAL Y MÉTODOS}

Se estudiaron prospectivamente 80 pacientes diagnosticados de tumor vesical, sometidos a
RTU y confirmados anatomopatológicamente como Ta, grados I-II, desde el 1/01/1998 hasta el $31 / 12 / 2000$. Estos pacientes se distribuyen aleatoriamente en 2 grupos de 40 para aplicar al primero de ellos (grupo I) Mitomicina-C endocavitaria a dosis de $40 \mathrm{mg}$ semanales durante 8 semanas y posteriormente otros $40 \mathrm{mg}$ mensuales durante un año; mientras que al segundo (grupo II) se le administró la misma terapia endovesical más Tegafur $800 \mathrm{mg}$ al día distribuidos en 2 tomas, durante un año. No se incluyeron los pacientes con mal estado general en los que se preveía que no se iba a poder completar el protocolo, los que tenían neoplasias asociadas, los que presentaban alteraciones de la función hepática, ni aquellos con edad mayor de 76 años.

Aparte de los estudios precisos para el diagnóstico y la categorización del tumor vesical, a todos los pacientes, y previamente al inicio del protocolo, se les realizó una evaluación bioquímica y hematológica, una analítica de orina y cultivo, un ECG, una placa de tórax y las primeras citología y cistoscopia a los 2 meses de la RTU. Estas 2 últimas exploraciones debían ser normales.

Tras el comienzo del tratamiento, todos los pacientes se sometieron trimestralmente a revisión clínica, estudio analítico sanguíneo, cistoscopia y citología de lavado hasta completar el primer año. Después, estos estudios se realizarian cada 6 meses salvo el caso de que el paciente presentase hematuria macroscópica. El seguimiento mínimo para poder ser evaluados se estableció en un año.

El estudio estadístico, en primer lugar, comparó las características antropométricas (edad, sexo) y las de los tumores (tamaño, número, primario o recidivado y grado) en ambos grupos. Las recidivas se compararon mediante su representación actuarial en una curva de Kaplan-Meier y su posterior análisis según los métodos de Greenwood y Willcoxon. El periodo libre de enfermedad se valoró mediante un test de MannWhitney. Por último se evaluaron los posibles efectos adversos tanto clínicos como analíticos.

\section{RESULTADOS}

El seguimiento fue similar en ambos grupos, diecinueve meses de media (intervalo: 13-34) en el grupo I y de 20,2 meses (intervalo: 12-36) en el 
grupo II. No se apreciaron diferencias significativas entre ambos grupos en su distribución por edades, (edad media grupo I: 64,2 ; grupo II: 62,6 ) y sexo (varones grupo I: $85,2 \%$; grupo II: $87,5 \%$ ). Tampoco se encontraron diferencias en las características tumorales (Tabla I).

\section{TABLA I}

CARACTERÍSTICAS DEL TUMOR

\begin{tabular}{|l|c|c|c||}
\hline & Grupo I & Grupo II & $\begin{array}{c}\text { Significación } \\
\text { estadística }\end{array}$ \\
\hline Únicos & 28 & 26 & NS \\
\hline Múltiples & 12 & 14 & NS \\
\hline$<3 \mathrm{~cm}$ & 25 & 27 & NS \\
\hline$>3 \mathrm{~cm}$ & 15 & 13 & NS \\
\hline GI & 9 & 10 & NS \\
\hline GII & 21 & 20 & NS \\
\hline Primario & 18 & 16 & NS \\
\hline Recidivado & 22 & 24 & NS \\
\hline
\end{tabular}

NS: no significativo.

La recidiva se produjo en 15 pacientes (37,5\%) del grupo I y $13(32,5 \%)$ del grupo II, no siendo significativa la diferencia (Tabla II). El periodo libre de enfermedad medio tanto para los que recidivan como para los que no fue de 27,3 meses para el grupo I y de 29,2 meses para el grupo II. El periodo libre de enfermedad medio solo en los pacientes que recidivaron fue de 13 meses en el grupo I, y de 15meses en el II. En ninguno de los casos la diferencia resultó significativa (Tabla II).

La Mitomicina no mostró mas efectos indeseables que una cistitis de pequeña intensidad en 2 casos del grupo I y un caso del grupo II. El Tegafur fue en general bien tolerado ya que solo se presentaron 2 casos de intolerancia gástrica (5\%), precisando uno de ellos suspender el tratamiento mientras que el otro solo tuvo que reducir

\section{TABLA II}

RESULTADOS GLOBALES

\begin{tabular}{|l|c|c|c|}
\hline & I & II & $\begin{array}{c}\text { Significación } \\
\text { estadística }\end{array}$ \\
\hline Recidivas & $15(37,5 \%)$ & $13(32,5 \%)$ & NS \\
\hline T.L.E. (meses) & 13 & 15 & NS \\
\hline
\end{tabular}

TLE: tiempo libre de enfermedad. NS: no significativo. la dosis a la mitad durante 4 meses, y un caso $(2,5 \%)$ de moderada y transitoria elevación de las transaminasas. No se encontró ninguna alteración hematológica o neurológica. Tampoco se apreciaron trastornos tróficos de piel o uñas. No apareció ningún caso de dermatitis.

\section{COMENTARIOS}

Los resultados de nuestro estudio permiten apreciar una tendencia a la menor recidiva y mayor periodo libre de enfermedad al asociar el Tegafur con la Mitomicina. Esta diferencia no resulta estadísticamente significativa por ser una muestra demasiado pequeña. De hecho, el estudio de Uyama ${ }^{6}$ de diseño similar a este pero con más de 100 pacientes, sí encontró diferencias estadísticamente significativas en el mismo sentido que nosotros. Un estudio más amplio, quizás con participación de varios centros, podría aumentar la potencia estadística y permitirnos sacar más firmes conclusiones. No hemos analizado el índice de recidivas separadamente por factores de riesgo (número de los tumores, grado, estadio, etc.) ya que reduciríamos aún más el número de pacientes por brazo no permitiendo sacar conclusiones.

Llama la atención al revisar los artículos publicados al respecto que hay dos grupos de estudios con resultados muy dispares; por un lado Lancina ${ }^{7}$ y Gelabert ${ }^{8}$, que, con un número de pacientes bajo ( 20 y 7 respectivamente) y un seguimiento corto (6 y 7 meses respectivamente), encuentran un índice de recidivas muy alto y una mala tolerancia al producto; y por otro lado los estudios de Unda $^{9}$, Moyano ${ }^{5}$, Kubota ${ }^{10}$, y Masuda $^{11}$, están más en la onda de nuestros resultados, con una mejora de la tasa de recidiva y una prolongación del tiempo libre de enfermedad, y una buena tolerancia general del Tegafur oral. El problema de la comparación de estas series es que los diseños son diferentes (Adriamicina vs Tegafur, BCG vs BCG más Tegafur, etc.).

Por nuestra parte podemos concluir que el Tegafur es un producto bien tolerado y seguro por vía oral, y que puede ser útil asociado a otros quimioterápicos intracavitarios en la profilaxis de la recidiva de los tumores superficiales de vejiga. 


\section{REFERENCIAS}

1. NEWLING DWW, HETHERIGTON J, SUNDARAN SK et al.: The use of valrubicin for the chemoresection of superficial bladder cancer- A marker lesion study. Eur Urol 2001; 39: 643-647.

2. KURTH KH.: Intravesical chemotherapy for superficial bladder tumors category Ta/T1: Who should be treated and how?. Semin Urol Oncol 1996; 14 (suppl 1): 30-35.

3. KURTH KH; PAGANO F, FAIR WR (eds).: Superficial bladder cancer, cap 4. Washington, Isis Medical Media, 1997.

4. VAN DER MEIJDEN APM, HALL RR, KURTH KH et al.: The marker tumor concept. Br J Urol 1996; 77: 634-637.

5. MOYANO CALVO JL, ROMERO DÍAZ JA, ORTIZ GÁMEZ A et al.: Estudio aleatorio prospectivo comparando BCG y BCG más Tegafur oral en la profilaxis de los tumores superficiales de vejiga estadio T1. Resultados a dos años y medio. Actas Urol Esp 1999; 23 (10): 843-847.

6. UYAMA T, AGA Y, YONEZAWA M.: Prophylactic longterm treatment of bladder tumors with oral chemotherapy (Tegafur). Urology 1984; 23 (4): 367-370.

7. LANCINA MARTÍN JA, GARCÍA PÉREZ M, ARRABAL MARTÍN M et al.: Quimioprofilaxis oral con Tegafur contra la recidiva de los tumores vesicales superficiales. Arch Esp de Urol 1988; 41: 135-138.
8. GELABERT MAS A, ARANGO TORO O, ROSALES BORDES A et al.: Citostáticos orales en la profilaxis del carcinoma de urotelio: nuestra experiencia. Arch Esp de Urol 1988; 41: 135-138.

9. UNDA URZÁIZ M, LIBANO ZUBIAUR C, PÉREZ FERNÁNDEZ A et al.: Adriamicina vs ftorafur como profilaxis en la recidiva en el carcinoma vesical superficial. Arch Esp de Urol 1990; 43 (2): 143-146.

10. KUBOTA Y, OSAKA M, FUKUSHIMA $S$ et al.: Prophylactic oral UFT therapy for superficial bladder cancer. Cancer 1993; 71: 1842-1845.

11. MASUDA F, MORI Y, TORII S et al.: A comparative clinical trial of UFT treatment and intravesical adriamycin instillation to prevent recurrence of superficial bladder cancer. Jpn J Cancer Chemother 1992; 19: 505-508.

Dr. G. Server Pastor

Servicio de Urología

Hospital Virgen de la Arrixaca

Ctra. Madrid-Cartagena, s/n

30120 El Palmar (Murcia)

(Trabajo recibido el 14 noviembre de 2001) 